\title{
Controversies about the management of invasive fungal infections in very low birth weight infants
}

\author{
Maria E. L. Moreira*
}

\begin{abstract}
Objective: This review encompasses the most recent publications about fungal infection in very low birthweight infants, keeping health professionals updated about this growing problem observed in neonatal units.

Sources of data: Original and review articles published over the past 15 years were searched in MEDLINE and Lilacs, using the following keywords: preterm infant, very low birthweight infants, sepsis, fungal infection, antifungal, Candida, amphotericin and fluconazole.

Summary of the findings: Invasive fungal infections affect especially preterm infants. Although new drugs (echinocandins) to treat fungal infection are available, amphotericin is the most widely used drug for the treatment of systemic candidiasis at this stage of life. Currently, there are four types of antifungal agents used in the treatment of fungal infections in neonates: polyene macrolides (amphotericin B deoxycholate and lipid preparations), azoles (triazoles), fluorinated pyrimidines (flucytosine) and echinocandins (caspofungin and micafungin). Two drugs capable of preventing invasive fungal infection are described: nystatin and fluconazole. The pharmacokinetics of fluconazole in the neonatal period is widely known and its prophylactic administration during the first six weeks of life has been associated with less invasive fungal infection in infants weighing less than $1,000 \mathrm{~g}$ at birth.

Conclusions: Fungal infections constitute an important problem in the neonatal intensive care unit and a better understanding of the incidence, diagnosis, clinical management, treatment, and prophylaxis is important in order to reduce morbidity and mortality. The identification of high-risk preterm infants and the implementation of prophylactic measures and early treatment may improve the outcome of these patients.
\end{abstract}

J Pediatr (Rio J). 2005;81(1 Suppl):S52-S58: Infection, very low birth weight infants, preterm infants, candida.

Fungal sepsis is becoming increasingly frequent in the neonatal period, especially among very low birthweight infants. Incidence is inversely related to gestational age and birthweight, ranging from $10 \%$ to $28 \%$ in infants weighing less than $1,000 \mathrm{~g} .{ }^{1}$ An investigation in about six neonatal units in the United States, conducted by the National Epidemiology of Mycosis Study Group over a period of two years, found candidemia to affect $1.2 \%$ of infants, out of which $82 \%$ had very low birthweight. ${ }^{2}$ Another study reported that, between 1981 and 1995, the number of cases of candidemia in the United States grew from $2.5 / 1,000$ to $28.5 / 1,000$, representing an 11 -fold increase. ${ }^{3}$

1. Researcher, Instituto Fernandes Figueiras - Fundação Oswaldo Cruz (IFF/FIOCRUZ), Rio de Janeiro, RJ, Brazil.

Suggested citation: Moreira ME. Controversies about the management of invasive fungal infections in very low birth weight infants. J Pediatr (Rio J). 2005;81(1 Suppl):S52-S58.
Most fungal infections in the neonatal period are caused by Candida albicans and Candida parapsilosis. Over the last years, the number of infections caused by other species, including Candida tropicalis, Candida glabrata, Candida krusei, Candida lusitaniae, and Candida guilliermondi has increased. In Brazil, infections caused by Candida parapsilosis were described both in very low birthweight infants and in adults. ${ }^{4}$ In São Paulo, some authors report Candida parapsilosis as the most frequently isolated species in blood cultures and intravascular catheters of children with candidemia. ${ }^{5}$

\section{Risk factors}

The following is a list by Saiman et al. ${ }^{2}$ of the main risk factors associated with systemic candidiasis, all of which must be considered in the prevention of the disease.

- gestational age < 32 weeks;

- previous fungal colonization (especially of the gastrointestinal tract); 
- presence of central venous catheters;

- prior use of parenteral nutrition and lipid emulsions;

- Apgar < 5 at five minutes;

- intubation time > 7 days;

- shock or coagulopathy.

One of the most important risk factors for invasive candidiasis is previous colonization by Candida. About $10 \%$ of premature infants will become colonized within the first week of life. The gastrointestinal tract is one of the first sites of fungal colonization. In a prospective study, Baley et al. ${ }^{6}$ found a $26.7 \%$ incidence of fungal colonization and a $7.7 \%$ incidence of systemic fungal disease in a total of 146 infants investigated. Colonization was predominantly observed in the gastrointestinal and respiratory tracts, during the first week of life. Saiman et al. ${ }^{7}$ in a prospective study involving 2,157 infants whose gastrointestinal tracts were cultured on admission and weekly until discharge found the rectum to be the most frequent site of colonization.

In both studies mentioned above, colonization of the gastrointestinal tract was associated with low gestational ages, higher SNAP scores (above 10), use of antibiotics especially third-generation cephalosporin -, presence of central venous catheter, and use of intravenous lipids and $\mathrm{H} 2$ blockers. ${ }^{6,7}$ Suppression of the normal gastrointestinal flora due to treatment with cephalosporins, especially thirdgeneration ones, and delayed enteral nutrition were associated with an increased colonization by Candida parapsilosis. $^{7}$

Early colonization is associated with Candida albicans and, although horizontal transmission can occur, vertical transmission seems to be a more common route of infection. ${ }^{6}$ Late colonization is associated with Candida parapsilosis, and horizontal transmission seems to be more common. ${ }^{7-9}$

\section{Systemic candidiasis}

Similarly to other forms of infection, the gold standard for diagnosing systemic candidiasis is the presence of Candida in samples of blood, urine, cerebrospinal fluid or any other sterile site in individuals with clinical signs of infection. Fungal infection is usually diagnosed around the third week of life. Saiman et al. ${ }^{2}$ investigating infants with an average birthweight of $1,187 \mathrm{~g}$ and a mean gestational age of 27.9 weeks found that the mean age at diagnosis was 22.9 days.

The clinical signs of systemic candidiasis are nonspecific and often undistinguishable from bacterial sepsis or other common findings of the neonatal period, such as hypothermia, gastric residue, hypoactivity, apnea, worsened respiratory function, hyperglycemia, hypotension, bradycardia, and abdominal distension. $3,6,10,11$ A rapid clinical worsening without an apparent cause, associated with the presence of risk factors, while using broad-spectrum antibiotics is suggestive of fungal infection. 12,13

Involvement of multiple organs is common among infants. Structures such as kidneys, meninges, eyes, bones and joints are variably involved in patients with candidemia and must, therefore, be systematically investigated. 10,12 Abdominal and cranial ultrasound, echocardiogram, and dilated fundus exam by an ophthalmologist should be requested whenever there is a suspicion of fungal infection. 13

Diagnosis of systemic candidiasis is not easy, which complicates the decision about when to start treatment. The mean time between a positive blood culture and the onset of treatment ranges from $2.1 \pm 1.3$ days to $5.1 \pm 3$ days, and several cases are diagnosed only at autopsy. ${ }^{2}$ Candida grows in several traditional culture media, but positivity rates, even in cases strongly suggestive of candidiasis, remain low. In premature infants, the sensitivity of blood cultures is also low. One of the factors that may affect this reduced sensitivity is the volume of blood collected, 14,15 since the chance of detecting a microorganism with $1 \mathrm{ml}$ of blood is $65 \%$ or less. ${ }^{15} \mathrm{~A}$ decreased concentration and a low rate of replication can reduce growth rates in culture media and increase the time needed to establish a positive diagnosis. $12,13,15$ Negative blood cultures, however, do not rule out the diagnosis of candidiasis and samples should, therefore, be serially collected.

Patients with findings suggestive of systemic candidiasis should be subjected to lumbar puncture for a complete cerebrospinal fluid analysis. A urine exam should also be requested for culture and direct examination. Urine samples should be collected with a bladder catheter or, preferably, by suprapubic puncture. ${ }^{6}$ A Gram stain showing the presence of yeast, either in cerebrospinal fluid or urine, is strongly suggestive of invasive candidiasis.

\section{Treatment}

Empirical treatment of infections by Candida in the neonatal period continues to be problematic, especially considering the toxicity of amphotericin. On the other hand, a delayed onset of treatment leads not only to an increased risk of mortality but also to higher rates of intraventricular hemorrhage, chronic pulmonary disease, retinopathy of prematurity, and developmental delay. Early treatment and removal of deep catheters are the best predictors of treatment success. ${ }^{16}$

Considering the time required to obtain positive culture results and the best prognosis associated with early treatment, Benjamin et al. ${ }^{17}$ argued that, in face of suggestive cases of fungal infection, empirical therapy should be started in late-onset neonatal sepsis, especially in the following situations:

- In all infants with less than 25 weeks of gestational age, regardless of the presence of thrombocytopenia.

- In infants with 25 to 27 weeks of gestational age who made use of third-generation cephalosporin or carbapenem up to 7 days before the presumed diagnosis, even in the absence of thrombocytopenia.

- In infants with unexplained thrombocytopenia, independently of gestational age. 
There are currently four classes of antifungal drugs available for the treatment of systemic candidiasis: polyenes, including amphotericin B (deoxycholate and lipid preparations), azoles (fluconazole and voriconazole), fluorinated pyrimidines (5- flucytosine), and echinocandins (caspofungin, micafungin). ${ }^{18}$

\section{Amphotericin}

Amphotericin B deoxycholate remains as the first-line drug of choice in the treatment of systemic candidiasis in infants. ${ }^{18,19}$ Amphotericin B is a macrocyclic polyene which acts by binding to ergosterol in the fungal cell membrane, increasing its permeability and leading to cell lysis and death. Amphotericin is not absorbed orally; therefore, it is only administered by the parenteral route, combined with deoxycholate, which improves its solubility in water. In the bloodstream, it dissociates from deoxycholate and binds to plasma proteins, being later distributed to tissues. ${ }^{18}$

The information available on the pharmacokinetics of amphotericin B is derived from small studies and presents extensive variability. Only two studies were published on the pharmacokinetics of amphotericin $B$, and both point to an extended serum half-life in infants when compared to adults. ${ }^{20,21}$ Most of the information comes from studies with older children and adults. Controlled clinical trials have not yet been published, and only retrospective analyses regarding the efficacy of treatment can be found in the literature. ${ }^{18}$

There is no consensus on the length of treatment. However, there is general agreement that one single Candida-positive blood culture constitutes sufficient evidence of infection and that the appropriate antifungal therapy should, then, be immediately started. Additionally, the need to remove existing central venous catheters until culture becomes negative is a well-established aspect of the therapy. ${ }^{19,22}$

In order to quickly achieve therapeutic levels, an immediate administration of 0.5 to $1 \mathrm{mg} / \mathrm{kg} /$ day of amphotericin B is currently recommended, since no response to the test dose has been observed in infants, who apparently tolerate the drug well. Amphotericin B should be diluted in a $5 \%$ glucose solution, with a concentration of $0.5 \mathrm{mg} / \mathrm{ml}$ for infusion via central catheter, or $0.1 \mathrm{mg} / \mathrm{ml}$ for infusion via peripheral veins. There are no advantages associated with a prolonged time of infusion. Short-term infusion increases drug availability by establishing a higher blood-tissue partition coefficient, without contributing to the frequency of adverse reactions. Amphotericin B does not need to be protected from fluorescent light. $23-26$

The main side effects of amphotericin B include nephrotoxicity, hepatotoxicity, myelotoxicity and, less frequently, cardiotoxicity. Adverse reactions have not been reported in infants. Nephrotoxicity is cumulative, induces systemic electrolytic alterations, and is characterized by renal tubular acidosis, hypokalemia and renal failure. Hypokalemia and hypomagnesemia are frequently observed in infants, requiring adequate supplementation. Anemia and thrombocytopenia may also accompany the use of amphotericin. Monitoring of urea, creatinine, magnesium, serum potassium, hematocrit and platelets is necessary during treatment. Some authors report that, due to a higher tolerance level, side effects in infants may be transient and less pronounced when compared to adults. 18,19,25

The new lipid formulations of amphotericin B were incorporated into the therapeutic arsenal with the goal of reducing side effects (especially nephrotoxicity), allowing higher drug dosages and an increased concentration in the reticuloendothelial system. Only two of the three existing formulations are available in Brazil: liposomal amphotericin $B$ and amphotericin B lipid complex. ${ }^{26}$ Information on the use of lipid formulations of amphotericin $B$ is derived from case reports. Scarcella et al. ${ }^{27}$ used liposomal amphotericin $B$ in 40 preterm and four full-term infants, finding a $72 \%$ efficacy. No side effects were observed. Five out of six patients with meningitis were cured. Walsh et al. ${ }^{28}$ used the amphotericin B lipid complex in 11 infants and also obtained satisfactory results, with minimal side effects. Linder et al. ${ }^{29}$ studied the tolerance and efficacy of three formulations of amphotericin in 56 infants with diagnosis of fungal sepsis. No differences were found in regard to efficacy. LopezSastre et al. ${ }^{30}$ recently published the results of a prospective study involving 118 infants with systemic candidiasis who received lipid formulations of amphotericin. The reported rate of mortality was $10.2 \%$, and all deaths occurred in very low birthweight infants. Costs for these new compounds are still very high.

The nature of the published studies on the use of lipid formulations does not allow for conclusions regarding their advantage when compared to amphotericin deoxycholate. It is not clear whether the differences observed in response to treatment are due to better tissue distribution or to higher dosages of amphotericin ( $1 \mathrm{mg}$ of deoxycholate amphotericin versus $5 \mathrm{mg}$ of lipid formulations). ${ }^{18}$ In addition, reports indicate that the pharmacological properties of lipid formulations reduce urinary drug excretion, compromising its efficacy in the treatment of urinary candidiasis. ${ }^{19,31}$ All authors agree on the need to perform randomized studies in infants comparing amphotericin B deoxycholate to lipid formulations. The standard dosage of lipid formulations ranges from 3 to $5 \mathrm{mg} / \mathrm{kg} /$ day. 18,19,26-30

The Infectious Diseases Society of America (2004) recommends that lipid formulations be considered as secondline drugs. They would be recommended in the following situations: 19

- unsuccessful treatment with traditional amphotericin B;

- patients with history of renal insufficiency;

- children with significant rise in creatinine levels during treatment (above $1.5 \mathrm{mg} / \mathrm{dl}$ ).

\section{Flucytosine}

Flucytosine, also known as 5-FC, is a fluorinated cytosine analogue, which is converted to fluorouracil by the body. Fluorouracil inhibits thymidylate synthetase, affecting fungal DNA and protein synthesis. The drug is well absorbed from the gastrointestinal tract and binds minimally to plasma 
proteins. Due to its synergism and high penetration in the central nervous system, flucytosine is used in association with amphotericin $B$, which independently of formulation, presents low penetration in the cerebrospinal fluid and central nervous system, even in the presence of meningeal inflammation. Flucytosine is available only for oral administration, which restricts its use in extremely preterm infants, who frequently do not tolerate oral drugs. Flucytosine is no longer available in Brazil.

\section{Azoles}

Fluconazole is a synthetic triazole compound, member of the azole family. Azoles act by inhibiting the enzyme responsible for the synthesis of ergosterol in the fungal cell membrane. Fluconazole is available for oral or intravenous administration, being well absorbed by the gastrointestinal tract. Fluctuation in gastric $\mathrm{pH}$ and presence of food do not modify drug absorption. Fluconazole shows low binding affinity for plasma proteins (12\%), being rapidly distributed to tissues, including the central nervous system. Elimination is predominantly renal. Hepatotoxicity is the most reported side effect; however, it is only observed in a small percentage of patients and resolves without the need to discontinue therapy. Fluconazole is not a fungicide. 18,26

Although the vast majority of studies on antifungal drugs in the neonatal period involve the use of fluconazole, there are still few well-controlled studies, defining dosage and ideal treatment length. ${ }^{18}$ Over the last years, several studies were published using fluconazole as monotherapy in infants with systemic candidiasis. Clinical efficacy was comparable to that of amphotericin B, with fewer side effects. The recommended dosage of fluconazole is $6 \mathrm{mg} / \mathrm{kg} / \mathrm{day}$, in a single dose, given orally or parenterally. ${ }^{18,19,32}$ The half-life of fluconazole in infants is long, but daily administration is, nevertheless, considered safe in very low birthweight infants with systemic candidiasis. ${ }^{19}$ The resistance of some non-albicans Candida species constitutes a limiting factor in the use of fluconazole.

Voriconazole is a new azole, a synthetic derivative of fluconazole, which also affects membrane integrity by inhibiting cytochrome $\mathrm{p}-450$ dependent enzymes needed for converting lanosterol to ergosterol. Voriconazole can work as a fungicide against some filamentous microorganisms and presents a wide spectrum of action against fluconazoleresistant species: Candida krusei and Candida glabrata. Voriconazole appears to be a promising drug for the treatment of candidiasis, but no reports of its use in infants have been published yet.

\section{Echinocandins}

Echinocandins are semi-synthetic derivatives of echinocandin B. They present a mechanism of action distinct from other antifungal agents, as they affect the fungal cell wall, instead of the cell membrane. Consequently, there are no effects on the cell membrane of the host, leading to a better drug tolerance. Information currently available suggests that echinocandins exhibit concentration- dependent activity. Caspofungin was the first echinocandin analogue made available. All species of Candida with clinical significance are sensitive to caspofungin, including those resistant to fluconazole. There is some controversy regarding its efficacy against Candida parapsilosis. Caspofungin is soluble in water and is incompatible with glucose solutions. There are no studies on the use of caspofungin in children or infants, but adult studies suggest that the drug is safe and, because of its antifungal action, may be more effective than azoles. This drug is available in Brazil, and its cost is extremely high. 18

\section{Current recommendations}

It is currently recommended that, in the presence of severe fungal infection, empirical treatment be started with amphotericin, until results of culture and sensitivity tests become available. After identification of the pathogen, the choice between fluconazole and amphotericin $B$ can be made based on the sensitivity profile. The identification of different species of Candida and the assessment of sensitivity to antifungal drugs are important to therapeutic success. Generally, Candida albicans, Candida parapsilosis, and Candida tropicalis should be treated with amphotericin B or fluconazole. Candida glabrata and Candida krusei show reduced sensitivity to fluconazole, so these cases should be treated with amphotericin B. Candida lusitaniae is resistant to amphotericin $B$, and fluconazole is the drug of choice. 19,26

Regarding the length of treatment, there is an ongoing debate surrounding all of the available drugs. The Infections Disease Society of America (IDSA) released a protocol with guidelines on the type of drug to be used according to the site of infection. This protocol was based on the available evidence and its recommendations for infants are summarized in Table $1 .{ }^{19}$

\section{Prophylaxis}

Prophylactic measures should be wide in range and scope. Strategies to reduce the incidence of colonization and, resultantly, of invasive disease - have been pursued. It is important to emphasize some guidelines for good practice in managing very low birthweight infants, which can contribute to a lower incidence of bacterial infection, 33 reducing the need for antibiotic therapy and, therefore, the incidence of invasive fungal infection. Potentially good practices that can reduce the number of new cases of infection in neonatal units are listed in Table 2.

Among the available antifungal agents, fluconazole is the most studied drug for prophylactic use. Availability of both parenteral and oral preparations and reduced toxicity are two of the factors contributing to this trend. However, there is still much controversy about which infants would benefit from its prophylactic use, especially when considering that fluconazole can be an important weapon against fungal sepsis affecting the central nervous system. Induction of resistance is a possibility to be considered, since all patients hospitalized in the same environment 
Table 1 - Guidelines for treatment of fungal infections in newborns (IDSA)

\begin{tabular}{ll}
\hline Fungal infections & Treatment \\
\hline Candidemia & $\begin{array}{l}\text { Therapy should be continued for } 2 \text { weeks } \\
\text { after the last positive blood culture result. } \\
\text { Amphotericin B may be switched to } \\
\text { fluconazole (intravenous or oral) for } \\
\text { completion of therapy, the choice depends } \\
\text { on the patient's sensibility. Removal of } \\
\text { existing intravascular catheters is } \\
\text { desirable. }\end{array}$
\end{tabular}

Congenital candidiasis Prematurely born neonates and neonates with low birth weight should be considered for systemic therapy: amphotericin B (10$25 \mathrm{mg} / \mathrm{kg}$ ) or fluconazole. In healthy full term infants with no evidence of candidemia, treatment with topical agents is generally appropriate.

Urinary candidiasis Candiduria must be taken into account in neonates with very low birth weight. The infection can be treated with amphotericin B for 7 - 14 days; removal of

Candidal meningitis Because of the tendency for this disease to

Candidal endocarditis Medical therapy with amphotericin B at

Candidal endophtalmitis

Candidal osteomyelitis and arthritis

would develop such resistance, and not only those who made use of fluconazole. This would ultimately limit the efficacy of fluconazole when needed. 35

Two prospective and randomized studies using intravenous fluconazole for prophylactic treatment of fungal infections were published.36,37 The prospective, randomized, placebo-controlled study by Kicklighter et al. 36 aimed at determining whether prophylactic fluconazole would reduce the incidence of candidal rectal colonization in very low birthweight infants. They found a $46 \%$ decrease in rectal colonization by Candida in the placebo group, against a decrease of $15.1 \%$ in the group treated with fluconazole. The drug was well tolerated in a $6 \mathrm{mg} / \mathrm{kg} /$ day dose. The only reported side effect was a slight elevation in hepatic enzymes, which normalized

Table 2 - Good practices to reduce fungal infection in neonates urinary catheters is often helpful. Fluconazole is recommended as well. Bladder irrigation with amphotericin $B$ is rarely indicated. relapse, therapy should be administered for a minimum of 4 weeks after resolution of all signs and symptoms associated with the infection. Amphotericin B $(0.7 \mathrm{mg} / \mathrm{kg}$ per day) plus flucytosine is appropriate as initial therapy. Prosthetic devices must be removed. maximal tolerated doses for 6 weeks has most often been used. Candida endocarditis has a propensity for relapse and requires careful follow-up for at least 1 year. Long-term therapy with fluconazole has been used after initial treatment. Clinical treatment, with no surgery, was shown to be effective in neonates with low birth weight. be continued until complete resolution of disease or stabilization. Courses of $6-12$ weeks of therapy are typically required. Fluconazole can be used to complete treatment if sensibility allows.

Surgical debridement and an initial course of amphotericin B for 2 - 3 weeks followed by fluconazole, for a total duration of therapy of 6 - 12 months, appear to be appropriate. Drainage and prolonged courses of therapy are required for arthritis management. The use of intra-articular therapy is discouraged.
Hand hygiene

Vascular line management

Rational use of antibiotics

Avoid petrolatum use

Early onset of enteral diet

Adequate hand hygiene of healthcare workers dealing with newborns is extremely important, specially when there are evidences of Candida transmission, particulary Candida parapsilosis, through the hands of those professionals. 2,7,9

Closed vascular systems implementation will avoid frequent changes and multiple connections. Use of appropriate antiseptic agents, with transparent dressing around the place of catheters insertion is recommended. Catheters should be removed as soon as there is suspicion of severe fungal infection that requires empirical antifungal medication. ${ }^{20,33}$

Adequate diagnostic tests will reduce the overuse of large spectrum antibiotics, specially cephalosporins, which is key in helping prevent fungal disease. $2,7,14$

Care with extreme premature infants' skin, avoiding abrasions resultant from dressings adhesion and venous punctures, will decrease the risk of invasive diseases. $7,34,35$

The goal is to reduce the enteral nutrition period, which is a proven risk factor of fungal sepsis incidence. Breast milk intake must be encouraged, as it contains candida antibodies. 7,35 .

Avoid $\mathrm{H} 2$ blockers

Gastric $\mathrm{pH}$ changes favors fungal colonization. $2,7,35$

Prophylactic use of drugs

The administration of endovenous fluconazole for 6 weeks in prematures with $<1,000 \mathrm{Kg}$ birth weight ( $3 \mathrm{mg} / \mathrm{kg} /$ day) in places of high fungal sepsis can decrease the incidence of invasive diseases. 36,37 
later. Fluconazole was used for four weeks, initially by intravenous route and later orally. One hundred and six infants participated in this study, which was not designed to show differences in the incidence of invasive disease, only in the reduction of colonization.

Kaufman et al. ${ }^{37}$ performed a prospective, double-blind, randomized trial using prophylactic fluconazole or placebo in the first six weeks of life of patients with birthweight below $1,000 \mathrm{~g}$. The aim of the study was to evaluate the efficacy of fluconazole in preventing fungal colonization and invasive infection by Candida. During the period of prophylactic use of intravenous fluconazole, fungal colonization was reported in $60 \%$ of infants in the placebo group and in $22 \%$ of infants in the fluconazole group. Invasive fungal infection with positive growth of fungal isolates from the blood, urine, or cerebrospinal fluid was documented in $20 \%$ of the patients in the placebo group, but in none of the 50 patients treated with fluconazole. Eight out of the 10 patients (80\%) who developed sepsis were colonized by the same species of Candida. Fluconazole was administered at $3 \mathrm{mg} / \mathrm{kg} / \mathrm{day}$ every third day for the first two weeks, every other day during the third and fourth weeks and daily in the last two weeks. No side effects were reported. Drug administration was discontinued if intravenous access was discontinued or if antifungal therapy was necessary for the treatment of a documented or presumed fungal infection.

A third study ${ }^{38}$, published only in the form of an abstract was included in the Cochrane Library systematic review ${ }^{39}$ of 2004. It focused on intravenous antifungal agents used in the prevention of systemic fungal disease. In the Cochrane meta-analysis, the three studies mentioned above were included, adding up to a total of 214 preterm infants. Reviewers concluded that there is enough evidence to support the claim that prophylactic fluconazole can contribute to reducing mortality rates due to systemic fungal infection. Nevertheless, they argued that the long-term consequences of this intervention are still to be determined. Multicenter studies involving a greater number of patients will be necessary in order to more clearly establish the risks and benefits associated with such strategy in low birthweight infants at risk for fungal infection.

The use of oral nystatin in the prevention of fungal colonization, with subsequent development of sepsis, is also being investigated. The Cochrane Library metaanalysis describes three articles using nystatin. ${ }^{40}$ The first compares it to placebo in a non-randomized trial, the second one compares nystatin to oral fluconazole, and the third one to miconazole. The authors concluded that there is not sufficient evidence to support the use of oral nystatin in the prevention of systemic fungal disease in intensive care units.

\section{Conclusions}

There remain a number of important controversies in the management of fungal colonization and sepsis in very low birthweight infants. However, there seems to be a general agreement on the use of amphotericin $B$ as the first-line drug to be used as empirical therapy. Length of treatment varies according to the site of the infection and it remains not well established. Prophylaxis with fluconazole can be an important strategy for reducing the incidence of systemic infection and mortality; however, this medication should be used with caution, especially because little is known on the possible development of drug resistance. Fungal colonization per se does not constitute an indication for prophylaxis and other factors must be considered. Infants weighing less than $1,000 \mathrm{~g}$ and using mechanical ventilation and central venous catheters can benefit from prophylactic drugs, especially in places where the incidence of fungal sepsis is high.

\section{References}

1. Baley JE, Ellis FJ. Neonatal candidiasis: ophthalmologic infection. Semin Perinatol. 2003;27:401-5.

2. Saiman L, Ludington $E$, Pfaller M. Risk factors for candidemia in Neonatal Intensive Care Unit patients. The National Epidemiology of Mycosis Survey Study Group. Pediatr Infect Dis J. 2000;19: 319-24.

3. Kossof EH, Buescher ES, Karlowicz G. Candidemia in a neonatal intensive care unit: trends during fifteen years and clinical features of 111 cases. Pediatr Infect Dis J. 1988;17:504-8.

4. Silva CL, Santos RM, Colombo AL. Cluster of Candida parapsilosis primary bloodstream infection in a neonatal intensive care unit. Braz J Infect Dis. 2001;5:32-6.

5. Matsumoto FE, Gandra RF, Ruiz LS, Auler ME, Marques SA, Pires $M F$, et al. Yeasts isolated from blood and catheter in children from a public hospital of São Paulo, Brazil. Mycopathologia. 2002;154:63-9.

6. Baley JE, Kliegman RM, Boxerbaum B, Fanaroff AA. Fungal colonization in the very low birth weight infant. Pediatrics. 1986;78:225-32.

7. Saiman L, Ludington E, Dawson JD, Patterson JE, RangelFrausto S, Wiblin RT, et al. National Epidemiology of Mycoses Study Group Risk factors for Candida species colonization of neonatal intensive care unit patients. Pediatr Infect Dis $\mathrm{J}$. 2001;20:1119-24.

8. Huang YC, Li CC, Lin TY, Lien RI, Chou YH, Wu JL, et al. Association of fungal colonization and invasive disease in very low birth weight infants. Pediatr Infect Dis J. 1998;17:819-22.

9. Lupetti A, Tavanti A, Davini P, Ghelardi E, Corsini V, Merusi I, et al. Horizontal transmission of Candida parapsilosis candidemia in a neonatal intensive care unit. J Clin Microbiol. 2002;40:2363-9.

10. Makhoul IR, Kassis I, Smolkin T, Tamir A, Sujov P. Review of 49 neonates with acquired fungal sepsis: further characterization. Review of 49 neonates with acquired fungal sepse: further characterization. Pediatrics. 2001;107:61-6.

11. Benjamin DK Jr, Ross K, McKinney RE Jr, Benjamin DK, Auten $R$, Fisher RG. When to suspect fungal infections in neonates: a clinical comparison of Candida albicans and Candida parapsilosis fungemia with coagulase-negative staphylococcal bacteremia. Pediatrics. 2000;106:712-18.

12. Chapman RL. Candida infections in the neonate. Curr Opin Pediatr. 2003;15:97-102.

13. Chapman RL, Faix RG. Invasive neonatal candidiasis: an overview. Semin Perinatol. 2003;27:352-6.

14. Benjamin DK Jr, Garges H, Steinbach WJ. Candida bloodstream infection in neonates Semin Perinatol. 2003;27:375-83.

15. Schelonka RL, Chai MK, Yoder BA, Hensley D, Brockett RM, Ascher DP. Volume of blood required to detect common neonatal pathogens. J Pediatr. 1996;129:275-8.

16. Kicklighter SD. Antifungal agents and fungal prophylaxis in the neonate. NeoReviews. 2002;3:e249-54.

17. Benjamin Jr DK, DeLong ER, Steinbach WJ, Cotton CM, Walsh TJ, Clark RH. Empirical therapy for neonatal candidemia in very low birth weight infants. Pediatrics. 2003;112:543-7.

18. Frattarelli DA, Reed MD, Giacoia GP, Aranda JV. Antifungals in systemic neonatal candidiasis. Drugs. 2004;64:949-68. 
19. Pappas PG, Rex JH, Sobel JD, Filler SG, Dismukes WE, Walsh TJ, et al. Infectious Diseases Society of America Guidelines for treatment of candidiasis. Clin Infect Dis. 2004;38:161-89.

20. Starke JR, Mason Jr EO, Kramer WG, Kaplan SL. Pharmacokinetics of amphotericin $B$ in infants and children. J Infect Dis. 1987; 155:766-74.

21. Baley JE, Meyers C, Kliegman RM, Jacobs MR, Blumer JL. Pharmacokinetics, outcome of treatment, and toxic effects of amphotericin B and 5-fluorocytosine in neonates J Pediatr. 1990;116:791-7.

22. Karlowicz MG, Hashimoto LN, Kelly Jr RE, Buescher ES. Should central venous catheters be removed as soon as candidemia is detected in neonates? Pediatrics. 2000;106:E63-8.

23. Correa AG, Baker CJ. Antifungal agents. In: Long SS, Pichering LK, Prober CJ, editors Principles and Practice of Pediatric Infectious Diseases. Philadelphia: Churchil Livingstone; 2003. p. 1511-1518.

24. Kintzel PE, Smith GH. Practical guidelines for preparing and administering amphotericin B. Am J Hosp Pharm. 1992;49: 1156-64.

25. Kingo AR, Smyth JA, Waisman D. Lack of evidence of amphotericin $B$ toxicity in very low birth weight infants treated for systemic candidiasis. Pediatr Infect Dis J. 1997;16:1002-3.

26. Campos JM, Menezes LF, Pone MV. Infecções fúngicas no período neonatal. In: Moreira ME, Lopes JM, De Carvalho M, editores. Recém-nascido de alto risco: teoria e prática do cuidar. Rio de Janeiro: Editora Fiocruz; 2004. p. 295-319.

27. Scarcella A, Pasquariello MB, Giugliano B, Vendemmia M, de Lucia A. Lipossomal amphotericin B treatment for neonatal fungal infections. Pediatr Infect Dis J. 1998;17:146-8.

28. Walsh TJ, Seibel NL, Arndt C, Harris RE, Dinubile MJ, Reboli A, et al. Amphotericin B lipid complex in pediatric patients with invasive fungal infections. Pediatr Infect Dis J. 1999;18:702-8.

29. Linder N, Klinger G, Shalit I, Levy I, Ashkenazi S, Haski G, et al. Treatment of candidaemia in premature infants: comparison of three amphotericin B preparations. J Antimicrob Chemother. 2003; 52:663-7.

30. Lopez Sastre JB, Coto Cotallo GD, Fernandez Colomer B, Grupo de Hospitales Castrillo. Neonatal invasive candidiasis: a prospective multicenter study of 118 cases. Am J Perinatol. 2003;20:153-63.

31. Agustin J, Lacson S, Raffalli J, Aguero-Rosenfeld ME, Wormser GP. Failure of a lipid amphotericin B preparation to eradicate candiduria: preliminary findings based on three cases. Clin Infect Dis. $1999 ; 29: 686-7$.
32. Huttova M, Hartmanova I, Kralinsky K, Filka J, Uher J, Kurak J, et al. Candida fungemia in neonates treated with Fluconazole: report of forty cases, including eight with meningitis. Pediatr Infect Dis J. 1998;17:1012-15.

33. Kilbride HW, Wirtschafter DD, Powers RJ, Sheehan MB. Implementation of evidence-based potentially better practices to decrease nosocomial infections. Pediatrics. 2003; 111:e519-33.

34. Campbell JR, Zaccaria E, Baker CJ. Systemic candidiasis in extremely low birth weight infants receiving topical petrolatum ointment for skin care: a case-control study. Pediatrics. 2000;105:1041-5.

35. Kaufman D. Strategies for prevention of neonatal invasive candidiasis. Semin Perinatol. 2003;27:414-24.

36. Kicklighter SD, Springer SC, Cox T, Hulsey TC, Turner RB. Fluconazole for prophylaxis against candidal rectal colonization in the very low birth weight infant Pediatrics. 2001;107:293-8.

37. Kaufman D, Boyle R, Hazen KC, Patrie JT, Robinson M, Donowitz LG. Fluconazole prophylaxis against fungal colonization and infection in preterm infants. N Engl J Med. 2001;345:1660-6.

38. Cabrera C, Frank M, Carter D, Bhatia J. Fluconazole prophylaxis against systemic candidiasis after colonization: a randomized. Double-blinded study. J Perinatol. 2002;22:604A.

39. McGuire W, Clerihew L, Austin N. Prophylactic intravenous antifungal agents to prevent mortality and morbidity in very low birth weight infants. Cochrane Database Syst Rev. 2004;(1):CD003850.

40. Austin NC, Darlow B. Prophylactic oral antifungal agents to prevent systemic candida infection in preterm infants. Cochrane Database Syst Rev. 2004;(1):CD003478.

Correspondence:

Maria Elisabeth Lopes Moreira

Departamento de Ensino

Instituto Fernandes Figueira

Av. Rui Barbosa, 716, Flamengo

CEP 22520-020 - Rio de Janeiro, RJ

Brazil 